\title{
COMMENT
}

\section{Continuous positive airway pressure and high flow nasal cannula: the search for effectiveness continues}

\author{
Hany Aly ${ }^{1}$ and Mohamed A. Mohamed ${ }^{2}$ \\ Pediatric Research (2020) 87:11-12; https://doi.org/10.1038/s41390-019-0626-y
}

In this issue, Kanbar et al. $^{1}$ prospectively studied the cardiorespiratory behavior of preterm infants while receiving continuous positive airway pressure (CPAP) and high flow nasal cannula (HFNC). At the time of extubation, mechanically ventilated premature infants with birth weight $<1250 \mathrm{~g}$ were randomly assigned to receive either one of the two modes. Crossover occurred after 45 min with infants who had received CPAP to be supported with HFNC and vice versa. The study did not find differences in cardiorespiratory parameters when alternating between the two non-invasive modalities. However, when receiving support via HFNC, infants experienced longer respiratory pauses (9.2 vs. $7.3 \mathrm{~s}$ ) and some of them developed bradycardia that required tactile stimulation. During this short period of monitoring, infants required significantly higher oxygen concentration while supported with HFNC when compared to the time of CPAP support. ${ }^{1}$

Respiratory support of premature infants has recently shown a significant shift towards non-invasive modalities. They have been used to facilitate the discontinuation of invasive mechanical ventilation as well as primary mode of support immediately after birth. ${ }^{2}$ HFNC is thought to work via washout of the dead space in the nasopharynx. ${ }^{2}$ In addition, gas flow via nasal cannula may provide a distending pressure. However, provided pressure may be inconsistent depending on multiple factors, including cannula caliber, infant weight, and gas flow rate. A recent study measured mean hypopharyngeal pressures of $2-6 \mathrm{cmH}_{2} \mathrm{O}$ when using gas flows of $0.5-3 \mathrm{~L} / \mathrm{min}$ via nasal cannula. However, peak pressures frequently measured $>15 \mathrm{cmH}_{2} \mathrm{O}$ when the flow rate was $2-3 \mathrm{~L} /$ min. ${ }^{3}$ Therefore, pressures delivered by nasal cannula with similar flow rates may be negligible or dangerously high. Furthermore, the current administration devices do not permit controlling or monitoring of the delivered pressure. The use of nasal cannula gained popularity in neonatal units mostly due to its convenient simplicity and therefore being preferred by nursing staff and parents. ${ }^{2}$

CPAP delivers more consistent pressures than HFNC and cannot exceed what is intended to be delivered. When provided to spontaneously breathing premature infants, CPAP pressure allows several physiologic benefits that include stimulating the respiratory drive splinting the airway and the diaphragm. Provided endexpiratory pressure maintains air sacs inflated, improves lung capacity, and decreases intrapulmonary ventilation-perfusion mismatch. ${ }^{4}$ These mechanisms may explain shortened respiratory pauses and decreased oxygen requirement during CPAP use that is reported in this issue.
Multiple clinical trials were conducted to assess the efficacy of CPAP as a primary mode for respiratory support in premature infants. The three largest trials included infants with gestational ages of 24-27 weeks (Surfactant Positive Airway Pressure and Pulse Oximetry Trial (SUPPORT) trial, $n=1316), 25-28$ weeks (CPAP or INtubation (COIN) trial, $n=610$ ), and 26-29 weeks (Dunn et al. trial, $n=648){ }^{5-7}$ Bronchopulmonary dysplasia (BPD) was identified when infants required oxygen support at 36 weeks of postconceptual age. When compared to mechanical ventilation, early use of CPAP was not associated with BPD reduction in the SUPPORT ( $40 \%$ vs. $44 \%)$, the COIN ( $29 \%$ vs. $35 \%)$, or Dunn et al. $(26 \%$ vs. $25 \%)$ trials. However, a meta-analysis including all CPAP trials reported a borderline reduction of $\mathrm{BPD}$ (relative risk $(\mathrm{RR})=$ 0.91; confidence interval (Cl): 0.81-1.01) and a significant reduction in the composite outcome of death or $\mathrm{BPD}(\mathrm{RR}=0.91$; $\mathrm{Cl}: 0.84-0.99)^{8}$

The use of HFNC as the primary mode of respiratory support was recently studied in randomized controlled trials in premature infants. A large multicenter trial recruited 583 premature infants with gestational age $\geq 28$ weeks aiming to compare HFNC to CPAP for early respiratory support without surfactant replacement therapy. Further recruitment for the study was stopped at the recommendation of the safety committee because of the significant inferiority of outcomes in infants who received HFNC. The rate of treatment failure was almost doubled with HFNC compared to CPAP $(25.5 \%$ vs. $13.3 \%, p<0.001) .{ }^{9}$ A more recent trial was conducted on premature infants with older gestational age $\geq 31$ weeks. The study recruited 754 infants and showed significant inferiority in outcomes when using HFNC. The treatment failure rate with HFNC was again double the CPAP failure rate $(20.5 \%$ vs. $10.2 \%, p<0.001){ }^{10}$ As HFNC is significantly inferior in premature infants $\geq 28$ weeks, it will be unlikely to conduct another trial with HFNC in the most vulnerable infants with gestational age $<28$ weeks.

Since nasal cannula is convenient to use by caregivers and comfortable for infants when attached to their nose, a newer version (RAM cannula, Neotech, Valencia, CA) that has a back hub to attach to the ventilator has been designed with the hope to deliver CPAP to the infant. However, this type of interface does not allow laminar gas flow since both inspiratory and expiratory gas meet in the common hub; turbulent gas flow typically creates high resistance. Studies on the use of RAM cannula showed high airway resistance and significant decrease in the delivered pressure. ${ }^{11}$ In addition, this type of NC has narrow lumens that are not suited to deliver well-heated and humidified gas, thereby exposing the

\footnotetext{
${ }^{1}$ Department of Neonatology, Cleveland Clinic Children's, Cleveland, OH, USA and ${ }^{2}$ Division of Newborn Services, The George Washington University Hospital, Washington, DC, USA

Correspondence: Hany Aly (alyh@ccf.org)
}

Received: 22 July 2019 Revised: 18 September 2019 Accepted: 7 October 2019

Published online: 22 October 2019 
premature infant's airway and lungs to a lower quality gas. If the gas flow is heated optimally to $37^{\circ} \mathrm{C}$ to provide $100 \%$ humidification, NC will have significant water condensations that will be delivered directly into the nares.

With bubble CPAP (b-CPAP), the inspiratory circuit transmits a constant gas flow to the patient and the distal end of the expiratory tube is immersed in a water bottle causing resistance and backward congestion that will transmit pressure to the patient. The study by Kanbar et al. ${ }^{1}$ utilized two different devices to deliver CPAP. Perhaps, the type of CPAP device did not matter in this 45-min physiology study. However, the assumption that different CPAP devices can produce similar clinical outcomes is unfounded. A randomized trial demonstrated better oxygenation for premature infants when receiving b-PAP as compared to their oxygenation while supported with ventilator-derived CPAP. ${ }^{12} \mathrm{~A}$ possible explanation for the better oxygenation may be the bubbles generated by gas flow under water create oscillations of the water level and pressure delivered to the patient. Therefore, the patient on b-CPAP is exposed to pressure oscillations around the desired pressure, rather than receiving a constant pressure. This oscillation effect may add to the efficacy of b-CPAP in volume recruitment. ${ }^{4}$ Anecdotally, centers that reported decreased incidence of BPD in very low birth weight infants to a singledigit figure have consistently used b-CPAP. ${ }^{13,14}$

The facial interface used to deliver CPAP is critical. The appropriate size of nasal prongs should fit without causing the nares to blanch and without surrounding gas leak. Prongs that are too small will create higher flow resistance and increased work of breathing. In addition, inappropriately small prongs will have a leak and consequently the intended pressure may not be delivered to the distal airways. Nasal prongs that are too large can get obstructed by the nasal alar cartilage and the pressure will not transmit through nasal passages. The shape of the nasal interface is important as well. Efficient nasal prongs should be curved to adapt to the anatomic shape of the internal nasal passage. As an alternative, facial masks are used to deliver CPAP. They can be useful in situations where the nasal openings are compromised to avoid irritation of skin and mucus membranes. However, the mask may not be as efficient as the prongs because it does not stent the nasal openings and gas leak can occur underneath the mask.

One of the interesting mechanisms of CPAP efficacy is its ability to stimulate lung growth. Neonatal ferrets exposed to CPAP were shown to have larger lung capacity and greater DNA and protein contents. Such effects were shown after 2 weeks of CPAP therapy, although they were not evident during the first week of therapy. ${ }^{15}$ Therefore, weaning an infant from CPAP to nasal cannula can potentially abort this process. A randomized controlled trial showed infants who were transitioned from CPAP to receive NC required longer duration of oxygen therapy and respiratory support. ${ }^{16}$ Neonatal units that report the lowest incidences of BPD advocate the use of CPAP for the entire respiratory support time rather than transitioning to NC. ${ }^{17}$ Thus, the successful use of CPAP is multifactorial requiring the use of appropriate size of prongs, anatomical shape of the nasal prongs, early application, and the devotion to CPAP therapy without alternating with NC. Together with other factors such as infant positioning, adequate and careful airway suctioning, and decompression of gastric air constitute the "b-CPAP bundle of care," which can lead to improved respiratory outcomes in premature infants. A checklist that details all elements of the b-CPAP bundle has been developed. ${ }^{17}$ It is important for future CPAP trials to specifically detail the type of CPAP device, the facial interface, and the bundle of care that are used during the trial. It is clear that variation of respiratory outcomes lies in these details.

CPAP care is laborious and requires time to build a team experience before achieving favorable outcomes. ${ }^{18}$ On the other hand, nasal cannula are user-friendly and is tempting for caregivers to apply to facilitate handling, kangaroo care, and oral feeds. However, the evidence to support its use and efficacy is lacking. It is fair to acknowledge that nasal cannula is an inferior therapy when compared to CPAP., ${ }^{9,10}$ Therefore, its use should be reserved to situations when CPAP is not feasible or is difficult to administer. Novel technologies to allow a more user-friendly CPAP device are needed.

\section{AUTHOR CONTRIBUTIONS}

H.A.: Developed the concept, drafted the manuscript, and approved its final version. M.A.M.: Contributed to the concept, revised the manuscript, and approved its final version.

\section{ADDITIONAL INFORMATION}

Competing interests: The authors declare no competing interests.

Publisher's note Springer Nature remains neutral with regard to jurisdictional claims in published maps and institutional affiliations.

\section{REFERENCES}

1. Kanbar L. et al. Cardiorespiratory behavior of preterm infants receiving continuous positive airway pressure and high flow nasal cannula post-extubation: randomized crossover study. Pediatr Res. (2019). https://doi.org/10.1038/s41390019-0494-5. [Epub ahead of print].

2. Manley, B. J. \& Owen, L. S. High-flow nasal cannula: mechanisms, evidence and recommendations. Semin. Fetal Neonatal Med. 21, 139-145 (2016).

3. González A. J. et al. Hypopharyngeal oxygen concentration and pressures delivered by low flow nasal cannula in preterm infants: relationship with flow, gas mixture, and infant's weight. Pediatr. Pulm. https://doi.org/10.1002/ppul.24441 (2019).

4. Aly, H., Mohamed, M. A. \& Wung, J. T. Surfactant and continuous positive airway pressure for the prevention of chronic lung disease: history, reality, and new challenges. Semin. Fetal Neonatal Med. 22, 348-53. (2017).

5. SUPPORT Study Group of the Eunice Kennedy Shriver NICHD Neonatal Research Network, Finer, N. N. et al. Early CPAP versus surfactant in extremely preterm infants. N. Engl. J. Med. 362, 1970-1979 (2010).

6. Morley, C. J. et al. Nasal CPAP or intubation at birth for very preterm infants. N. Engl. J. Med. 358, 700-708 (2008).

7. Dunn, M. S. et al. Randomized trial comparing 3 approaches to the initial respiratory management of preterm neonates. Pediatrics 128, e1069-e1076 (2011).

8. Schmölzer, G. M. et al. Non-invasive versus invasive respiratory support in preterm infants at birth: systematic review and meta-analysis. BMJ 347, f5980 (2013); erratum in BMJ 348,g58 (2014).

9. Roberts, C. T. et al., HIPSTER Trial Investigators. Nasal high-flow therapy for primary respiratory support in preterm infants. N. Engl. J. Med. 375, 1142-1151 (2016)..

10. Manley, B. J. et al., HUNTER Trial Investigators. Nasal high-flow therapy for newborn infants in special care nurseries. N. Engl. J. Med. 380, 2031-2040 (2019)..

11. Green, E. A., Dawson, J. A., Davis, P. G., De Paoli, A. G. \& Roberts, C. T. Assessment of resistance of nasal continuous positive airway pressure interfaces. Arch. Dis. Child Fetal Neonatal Ed. https://doi.org/10.1136/archdischild-2018-315838 (2018).

12. Courtney, S. E., Kahn, D. J., Singh, R. \& Habib, R. H. Bubble and ventilator-derived nasal continuous positive airway pressure in premature infants: work of breathing and gas exchange. J. Perinatol. 31, 44-50 (2011).

13. Aly, H., Massaro, A. N., Patel, K. \& El-Mohandes, A. A. Is it safer to intubate premature infants in the delivery room? Pediatrics 115, 1660-1665 (2005).

14. Van Marter, L. J. et al. Do clinical markers of barotrauma and oxygen toxicity explain interhospital variation in rates of chronic lung disease? The Neonatology Committee for the Developmental Network. Pediatrics 105, 1194-1201 (2000).

15. Zhang, S., Garbutt, V. \& McBride, J. T. Strain-induced growth of the immature lung. J. Appl. Physiol. 81, 1471-1476 (1996).

16. Abdel-Hady, H., Shouman, B. \& Aly, H. Early weaning from CPAP to high flow nasal cannula in preterm infants is associated with prolonged oxygen requirement: a randomized controlled trial. Early Hum. Dev. 87, 205-208 (2011).

17. Aly, H. \& Mohamed, M. A. In Atlas of Procedures in Neonatology 6th edn (eds Ramasethu, J. S. \& MacDonald, M. G.) (Lippincott, Williams \& Wilkins, 2019).

18. Aly, H., Milner, J. D., Patel, K. \& El-Mohandes, A. A. Does the experience with the use of nasal continuous positive airway pressure improve over time in extremely low birth weight infants? Pediatrics 114, 697-702 (2004). 